\title{
Die Informalisierung der Textil- und Bekleidungserzeugung am historischen und aktuellen osteuropäischen Beispiel
}

Statistiken über die Anteile des informellen Sektors ${ }^{1}$ an Gesamtbeschäftigung und Wirtschaftsleistung belegen zwei Tatsachen: Erstens war und ist die „Schattenwirtschaft“ nicht regional oder zeitlich begrenzt. Zum zweiten nimmt sie in den letzten Jahren weltweit zu. Auch in Deutschland ist der Anteil der "Schattenwirtschaft" am Bruttoinlandprodukt (BIP) innerhalb der letzten 20 Jahre von 6\% auf 15\% angestiegen (ILO 1998a: 28). Die Mehrheit der informell Beschäftigten sind Frauen. Ihr Anteil liegt bei ca. $60 \%$ und variiert erstaunlicherweise wenig zwischen Industrie- und sogenannten Entwicklungsländern (Morgenrath/Wick 1996: 18).

Die „Schattenwirtschaft“ liegt im Trend der globalen Deregulierung. Sie wird als flexibilisierte, abrufbare Zulieferwirtschaft und Vertragsarbeit hoffähig und als Jobwunder instrumentalisiert. Höchste Zeit, die ÜberlebenskünstlerInnen aus ihrem Schattendasein herauszuholen, in dem sie trotz vieler engagierter Vorstöße von Wissenschaftlerinnen und Aktivistinnen immer noch sind.

Das Wachstum des informellen Sektors scheint der Meinung recht zu geben, daß es sich um ein temporäres Problem von ungenügender Qualifikation und/oder temporärem Arbeitsplatzmangel im formellen Bereich handelt (z.B. bei ILO 1998: 21). Aus historischer wie auch aktueller Perspektive soll dies am Beispiel des Bekleidungssektors hinterfragt und gezeigt werden, daß formelle und informelle Arbeit zwei Seiten des kapitalistisch rationalen Arbeitsmarktes sind.

1 Arbeitsdefinition: Informelle Arbeiten sind ganz „,normale“, legale wirtschaftliche Tätigkeiten, die allerdings unter den Bedingungen der teilweisen oder vollständigen Abwesenheit rechtlicher und sozialer Absicherung ausgeübt werden bzw. nur bei Umgehung dieser Absicherung ausgeübt werden können, weil der formelle Sektor protektionistisch 'abgeschottet' ist. Subsistenzarbeit wird i.A. nicht dazu gezählt. 


\section{Die Rolle informeller Arbeit bei der Entstehung der industriellen Textilbranche und regulierende Reformansätze}

Die Textil- und Bekleidungsindustrie wird nach wie vor als Schwelle und Einstiegsbranche in die industrialisierte kapitalistische Entwicklung angesehen. Von ihr sollte dann ein Trickle Down-Effekt für die gesamte Wirtschaft, eine Art Initialzündung, ausgehen. Für sogenannte Entwicklungsländer wurden diese Effekte abgesehen von Teilwirkungen nicht erreicht, und sie sind wohl auch nicht erreichbar (Köpke 1998: 11ff, 90ff, Altenburg/Walker 1995: 102-112, Wick 1998: 246). Dagegen ist die historische Rolle dieser Branche unbestritten. Weniger beachtet wurde jedoch die Bedeutung, die protoindustrielle, informelle Arbeitsformen dabei hatten. Diese wird im folgenden zunächst historisch skizziert, um dann Parallelen zur gegenwärtigen Situation in der Bekleidungsindustrie aufzuzeigen.

Die ländliche Haus-Protoindustrie, die überwiegend Textilien verarbeitete, schuf während ihrer Blüte im 18. Jh. einige der Voraussetzungen für ein kapitalistisches Akkumulationsregime. Der Kern dieser Hausindustrie war der Haushalt als „Produktionseinheit“ ohne klare Abgrenzung zwischen Markt- und Eigenbedarfsproduktion (Komlosy 1997: 66). Das entsprach den vorgefundenen feudalen, bäuerlichen bzw. ständischen Gesellschaften, die von einem komplexen Regelsystem stabilisiert wurden. Diese vorindustriellen Formalia behinderten jedoch die Expansion der nichtzünftischen Unternehmen. Infolgedessen wurde das feudale und zünftische Regelwerk zunehmend außer Kraft gesetzt bzw. umgangen. Die Zünfte ihrerseits gerieten durch diese Unternehmen unter Kostendruck. Die oft durch kapitalkräftige städtische Großhändler betriebene ländlich angesiedelte Fertigung war meist kostengünstiger als die städtische Handwerksproduktion. In ländlichen Gebieten gab es billige Arbeitskräfte in großer Zahl. Zudem waren städtische Räume effektiver zünftisch durchreguliert, so daß Arbeitskräfte dort schwer mobilisiert werden konnten. Zur Verfügbarkeit von Arbeitskräften mußte die Verfügbarkeit von Rohstoffen und Absatzmärkten kommen, die der koloniale Dreieckshandel lieferte - eine Vorform des global sourcing.

Das vorindustrielle Reglement konnte durchgreifend nur durch eine neue staatliche Wirtschaftsförderung und -politik außer Kraft gesetzt werden. Sie sicherte die Mobilität der Produktionsfaktoren und Erzeugnisse - „Protoindustrialisierung als Deregulierung“ (Komlosy 1997: 68/69). Der entstehende Zentralstaat half intensiv bei der Rekrutierung der Arbeitskräfte, indem er z.B. die „Familien- und Hausstandsgründung“ für ländliche Unterschichten durch Ausweisung entsprechender Ansiedlungsflächen förderte. Dabei enthielten die Parzellen ganz bewußt ein kleines Stück Land für die Eigenversorgung. Diese Wirtschaftsförderung sah auch Maßnahmen zur Be- 
strafung von „Müßiggängern“ vor, die sich für das textile Verlagswesen nicht verfügbar halten wollten. Ohne die Umgehung und Abwesenheit von kostspieligen Regeln in der Hausindustrie des textilen Verlagswesens wäre keine Fabrik entstanden, keine jener „Enklaven kapitalistischer Produktion (...), deren Logik in der Folge die gesamte Weltwirtschaft beherrschen sollte“ (ebd.: 65). Zum Bestandteil dieser Logik wurde es, „,die Ausnahme zur Norm“ zu erklären (ebd.: 64). Nicht die kapitalistische Großfabrik, sondern der auf ländlicher Hausindustrie basierende Verlags-Großbetrieb bildete den Prototyp der kapitalistischen Wirtschaftsweise bereits vor der Mechanisierung in der Textilerzeugung.

Der oder die aus feudaler Abhängigkeit entlassene Landlose fand im textilen Verlagswesen eine Möglichkeit, Einkommen und damit das Überleben zu sichern. Deregulierung feudaler Abhängigkeiten und Schranken erzeugte einen Dualismus: eine Konservierung ländlicher Produktionsformen der ungeregelten und subsistenzwirtschaftlichen Arbeit sowie Inseln geregelter Arbeit in den Manufakturzentren. Einerseits also - ausgedrückt in heutiger Terminologie - ausgelagerte, verlängerte Werkbänke für arbeitsintensive Teile der textilen Kette, die tendenziell informalisiert werden, andererseits Zentralisierung von Management, Design, Qualitätssicherung, Beschaffung und Vermarktung in geregelten Arbeitsverhältnissen. Die kapitalistisch rational gestaltete Koexistenz zwischen informeller und formeller Lohnarbeit war geboren. Für sie waren und sind textile Verarbeitungsgänge wie geschaffen.

Der Dualismus in den Produktionsformen drückte sich im strukturell heterogenen Charakter der Heimindustrie vor der Mechanisierung der Textilund Bekleidungserzeugung aus: Zum einen herrschten aufgrund der Eigenversorgung bäuerliche Beziehungen vor, in denen der Haushalt als „Lebens- und Produktionsgemeinschaft" (Ulla Knapp) nach wie vor dominierte. Zum anderen waren die AkteurInnen aus der feudalen in die Marktabhängigkeit entlassen worden. Diese strukturelle Heterogenität hat sich aus schlichter Existenznot bis heute erhalten: So überleben osteuropäische NäherInnen-Familien nur durch Teilnahme an der Subsistenzwirtschaft von Teilen der Großfamilie auf dem Lande.

Die damals positive Seite dieser Heterogenität: Mit dem eigenen Stückchen Land und dem eigenen Einkommen war die Möglichkeit sozialen Aufstiegs verbunden. Sie findet gegenwärtig eine gewisse Parallele im neuen Selbstbewußtsein vieler Näherinnen in Bangladesh oder Honduras, die erstmalig der patriarchalen Großfamilie entkamen und ein eigenes Einkommen erhalten (vgl. Dannecker 1998, Köpke 1998: 169).

Ulla Knapp wies auf eine bemerkenswerte, mit diesem sozialen Aufstieg verbundene Entwicklung in der hausindustriellen geschlechtsspezifischen Arbeitsteilung hin (Knapp 1984: Abschnitte 2.2., 2.3.): Die wenigen Er- 
werbsmöglichkeiten für die ländlichen Unterschichten nach Lockerung der feudalen Bindungen erforderten eine relativ hohe Flexibilität in der innerfamiliären Arbeitsteilung. Bei der Aufteilung von Familien-, Subsistenzund Lohnarbeit wurde die bürgerliche wie auch die bäuerlich-patriarchale Zuweisung der unbezahlten Reproduktions- und Subsistenzarbeit an die Frauen teilweise aufgebrochen - zugunsten des maximalen Gesamtfamilieneinkommens (vgl. auch Komlosy 1997: 76/77). Auch die traditionellen Zuweisungen des Spinnens an ,geschickte' Frauenarbeit und des Webens an Männer konnten unter diesen Bedingungen nicht aufrecht erhalten werden. Dem Patriarchat war die materielle Basis (Eigentum und feudale Privilegien) entzogen, was zu einer gewissen wirtschaftlichen Egalität der Geschlechter in Produktions-, Konsum-, Sexual- und politischen Beziehungen führte. Die Antwort der bürgerlichen Öffentlichkeit ließ nicht auf sich warten: Den Heimarbeiterinnen wurde Sittenlosigkeit, Faulheit und Gottlosigkeit vorgeworfen.

Auch in diesem Zusammenhang drängen sich Parallelen zur Situation asiatischer oder zentralamerikanischer Näherinnen auf: Ihre Abwesenheit von der ländlich-patriarchalen Großfamilie wird als sittliche Gefahr und als Freibrief für unwürdige Behandlung und Gewalt ausgelegt. Die häusliche und außerhäusliche Gewalt (z.B. auf den Arbeitswegen) an Arbeiterinnen nimmt zu, gerade weil sie mehr Selbstbewußtsein und Unabhängigkeit gegenüber den traditionellen Familienrollen zeigen; es findet eine „Modernisierung der Gewaltverhältnisse“" statt (vgl. Bickham Mendez/Köpke 1999, Da Bindu Collective 1998).

Der relative emanzipatorische Spielraum innerhalb der Hausindustrie vor der Mechanisierung der Textil- (Web- und Spinnmaschine) und der Bekleidungserzeugung (Nähmaschine) basierte also auf der Demontage feudaler, ständischer Regeln. Die Abwesenheit dieser Regeln egalisierte zunächst die Beziehungen zwischen den Geschlechtern in der HeimarbeiterIn-Familie. Gleichwohl profitierte der Textilkapitalist gerade davon, denn er hatte es nicht nötig, HeimarbeiterInnen adäquat zu entlohnen. Er setzte voraus, daß sie sich selbst versorgten, flexibel auf die Schwankungen der Auftragslage reagierten und weitere Einkommens- und Versorgungsquellen hatten. Diese Argumentation wird auch heute gegenüber ungeregelten Arbeitsformen im Bekleidungsgewerbe benutzt.

Ulla Knapp (1984: Abschnitt 2.2.) weist auf einen weiteren Grund der Unterbezahlung hin: der Ausschluß von Frauen aus dem vorindustriellen Handwerk und den Zünften. Da sie nicht privilegiertes Zunftmitglied werden konnten, drängten sie in wenig professionalisierte, ungeregelte, instabile Tätigkeiten z.B. als Tagelöhnerinnen und Heimarbeiterinnen. Damit war die Unterbezahlung weiblicher Lohnarbeit, verbunden mit der Ausnutzung unbezahlter Reproduktions- und Subsistenzarbeit, konstituiert. 
Die Qualifikationen von Frauen waren und sind gerade im Textil- und Bekleidungsgewerbe nicht formalisiert, gleichwohl im Produktionsprozeß gefragt. Die berühmte, Frauen angeblich angeborene Fingerfertigkeit, zwar explizit damals wie heute als besonderer Vorteil von Frauenarbeit betont, wird jedoch nicht entlohnt. Sie kommt in keinem Berufsbildungsregister vor. Ein anderes Beispiel: Fragt man heute in Nähfabriken, warum Frauen bügeln - eine der am schlechtesten bezahlten Tätigkeiten -, heißt es, es sei eine Arbeit, die keine Qualifikation erfordere. Bügeln ist keine formalisierte berufsbildungsmäßig erworbene Fertigkeit, sondern eine in jahrelanger unbezahlter Hausarbeit anerzogene. Wenn Männer bügeln, heißt es, die Arbeit sei so schwer, weshalb Erschwerniszulagen nötig seien.

Die textile Hausindustrie ermöglichte - neben anderen Bedingungen - die industrielle Entwicklung, indem Werte abgezogen wurden (Arbeitsrente), die aus der Unterbezahlung der (Heim-)Lohnarbeit und der Ausnutzung unbezahlter Eigenversorgung resultierten. Die Wohnstuben und Häuser der Hausindustriellen dienten als kostenlose Produktionsgebäude, die gesamte Familie einschließlich der Kinder kooperierte für den (Neben-)Erwerb, die landwirtschaftliche Eigenversorgung ermöglichte Lohneinsparungen. Zweck informalisierter Arbeit ist also in erster Linie ihre Unterbezahlung. Die verbreitete Diagnose mangelnder Qualifikation, sofern sie überhaupt den Tatsachen entspricht, bezieht sich auf marktförmige, aus der formellen Berufsbildung abgeleitete Bildungsstandards.

\section{Die Heimindustrie nach Einführung der Web-, Spinn- und Nähmaschine}

Im 19. Jh. wurden Teile der Textil- und Bekleidungsfertigung mechanisiert (Weben, Spinnen, Nähen). Die Näherei entwickelte sich zur Domäne der städtischen Heimarbeit. Mit der Mechanisierung und der so ermöglichten Expansion der Fabrik veränderte sich auch die Funktion und der Charakter der Heimindustrie: Ihr gestalterischer Spielraum wurde wesentlich eingeengt, denn sie wurde zum billigen, flexiblen, abrufbaren Anhängsel der Fabrik. Die egalitäre und arbeitsteilige Haushaltsführung vorindustrieller, semiproletarischer Familien war damit obsolet. Produktionsvor- und nachbereitende Tätigkeiten sowie noch nicht mechanisierte Arbeitsgänge wurden in Heimarbeit gegeben, die dadurch gänzlich von den Ansprüchen, dem Takt der Maschinen und der Organisation der fabrikmäßigen Produktion abhing. Heimarbeit in Form der ländlichen Textilverarbeitung und der städtischen Maschinennäherei bildete nunmehr die Reserve und das Auffangbecken für die Fabrik. Dort fanden die durch Fabrikarbeit verschlissenen wie auch die aus dem Niedergang der alten Hausindustrie und des Gewerbes entlassenen Arbeitskräfte ein wenn auch miserables Einkommen. 
Eigenversorgung war kaum noch möglich, weil die ländlichen HeimarbeiterInnen ihr Stück Land meist verloren bzw. die städtischen Heimnäherinnen nie Land besessen hatten. Die Fabrik reproduzierte sich ihre flexible, billige ,verlängerte Werkbank“ ständig neu.

„Was als sozialer Aufstieg begonnen hatte, führte unter veränderten ökonomischen und technologischen Rahmenbedingungen zur besonderen Verwundbarkeit der Heimarbeit“" (Komlosy 1997: 76). Ungeregelte Arbeit wurde allmählich in den Schatten der proletarisierten Inseln ins Haus, ins Private verwiesen und ,,von der Öffentlichkeit immer weniger als gesellschaftlich notwendige Arbeit anerkannt" (ebd.: 65). Solcherart degradierte Arbeit war folgerichtig un- oder unterbezahlte Arbeit. Jede Arbeit, die

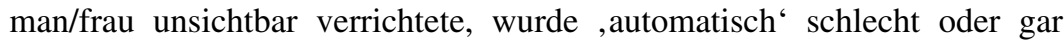
nicht bezahlt und geschätzt. Dagegen rückten die proletarisierten männlichen „Helden der Arbeit“ (Ingrid Kurz-Scherf) ins Zentrum der Aufmerksamkeit der entstehenden Sozialdemokratie und Gewerkschaften, wo sie nach wie vor stehen. Erst durch diese Gegenüberstellung zur , eigentlichen ", geregelten Arbeit und durch die Anhängselfunktion wurde die informelle Arbeit sozial geschlossen, stigmatisiert, in den ,Schatten' gestellt.

In jahrzehntelangen gesellschaftlichen Auseinandersetzungen (z.B. gegen unmenschliche, ausbeuterische Kinderarbeit in Fabriken) ist der männliche Fabrikarbeiter inthronisiert worden. Sein Status wurde durch Sozialgesetzgebung (vgl. Hausen 1997) und später durch den fordistischen Gesellschaftsvertrag in umfangreichen Regelwerken und Verhaltensweisen gesichert. Die Kehrseite war die Marginalisierung nicht geregelter, meist weiblicher Arbeit, die weiteren Wertabzug ermöglichte: ein Teufelskreis, dem nur noch mit einer weitere Ausweitung der Arbeitszeit, mit informellen Netzen und flexibler Zähigkeit begegnet werden konnte (vgl. Komlosy 1997: 77). Auch das sind Strategien, wie sie im heutigen informellen Sektor praktiziert werden (müssen).

Der Prozeß sozialer Schließung in der Heimnäherei des 19. Jh. ging einher mit geschlechtsspezifischer Segregation. Die „bürgerlich-kapitalistische Erfindung“ von der „Ernährerrolle des Mannes“ (Knapp) setzte sich als gesellschaftliche Norm durch. So unterstellen Erhebungen in der Heimindustrie jener Zeit, daß Haushaltsvorstand und Ernährer der Vater/Mann ist und die Mutter/Frau im dem Mann untergeordneten Nebenerwerb arbeitet. Ihre Familien-, Reproduktions- und Subsistenzarbeit ist keiner Erwähnung wert (Hausen 1986: 10ff, Komlosy 1997: 79). Heimnäherinnenarbeit wird als vorübergehend und nur zuverdienend angesehen neben der, eigentlichen Bestimmung der Frauen - ein Freibrief für intensivste Ausbeutung. Bis heute werden Frauen in teilweise oder vollständig informalisierten Konfektionsarbeiten mit dieser Legitimation ausgenutzt unter Einschränkungen ihrer persönlichen Würde und sexuellen Selbstbestimmung. 


\section{Strategien zur Regulierung der Hausarbeit}

Während der Jahrhundertwende und bis in die 20er Jahre hinein gab es eine erstaunlich breit geführte gesellschaftliche Debatte um die Heimarbeit und ihre Regulierung (vgl. Komlosy 77ff, Hausen 1986). Darin lassen sich Argumentationsmuster der Stigmatisierung und geschlechtsspezifischen $\mathrm{Zu}-$ richtung von Heim-Lohnarbeit bzw. Fabrikarbeit erkennen. Diese Argumentationen spielen nach wie vor eine Rolle und sind für die gegenwärtige Diskussion um Regulierungsalternativen sehr aufschlußreich. Als ein Kontinuum vieler sozialreformistischer Bestrebungen erwies sich die einseitige Konzentration auf die Fabrik als dem rationellen Paradestück des Kapitalismus, in der Frauenarbeit die Ausnahme von der Regel darstellt.

Durch den Druck von ArbeiterInnen-, christlichen und bürgerlichen Bewegungen wurden die industriellen Beziehungen in den Fabriken des vorigen Jahrhunderts technisch und sozial modernisiert. Dagegen drückten viele Sozialreformer der ungeregelten Heimlohnarbeit den Stempel „empörendster Verhältnisse“ (Kautsky) auf. Marxismus, Gewerkschaften und Sozialdemokratie konzentrierten sich auf die Fabrik und erachteten ungeregelte Arbeit als temporären Auswuchs kapitalistischer Ausbeutung. Durch Mindestlohngesetze z.B. müsse man solche Formen der Überausbeutung bekämpfen, um sie kostenmäßig unattraktiv zu machen und in die effizientere Fabrik eingehen zu lassen. HeimarbeiterInnen sollten zu klassenbewußten FabrikarbeiterInnen werden. Produktivitätserhöhung und Fortschritt im Kapitalismus führten nach dieser Auffassung unweigerlich zur Beseitigung solcher ,Vorformen wirklich kapitalistischer' Produktion (vgl. Komlosy 1997: 79, Hausen 1986: 2/3). Heim-Lohnarbeit wurde in der Tat verdrängt, nur um in noch weniger sichtbare Bereiche der Peripherie in den industrialisierten Ländern selbst ${ }^{2}$ und in die Kolonien zu verschwinden. Sie blieb ein Strukturelement kapitalistisch rationaler Organisation der Textil- und Bekleidungserzeugung in globalem Rahmen. Ungeregelte Arbeit ist also keineswegs unrationell. Im Marxismus ist eine Relativierung des linearen Forschrittsdeterminismus angelegt, nach der Produktivität kein Selbstzweck, sondern kapitalistischer Rationalität untergeordnet ist. Gerade diese Rationalität begründet die Koexistenz geregelter neben wirtschaftlich und sozial diskriminierter und damit billiger, williger, insbesondere weiblicher Lohnarbeit im informellen Sektor.

Auch heute wird von Gewerkschaften oft argumentiert, daß ein Engagement für informelle ArbeiterInnen sich nicht lohne, weil diese Tätigkeiten sowieso früher oder später mechanisiert oder automatisiert, jedenfalls wegrationalisiert würden. Nach wie vor setzen Gewerkschaften, soweit sie den

2 Nach wie vor gibt es beispielsweise Heimnäherinnen auf der Schwäbischen Alb. Sie nähen u.a. für Unternehmen, die sich mit dem Festhalten am „Standort“ rühmen. 
informellen Sektor überhaupt beachten, auf rigorose Formalisierung unabhängig von den konkreten Bedingungen und Bedürfnissen der dereguliert Beschäftigten, besonders der Frauen. Arbeiterinnen kann z.B. die Forderung nach Trinkwasser wichtiger sein als das gewerkschaftliche Organisationsrecht (vgl. Bickham Mendez/Köpke 1999). Der doppelte Druck, dem sich Frauen ausgesetzt sehen, sowohl Geld verdienen als auch die Familie/den Haushalt besorgen zu müssen, wird unterschätzt. Das zwang und zwingt sie zur Aufnahme schlechtester Jobs bei Untergrabung ihrer eigenen Gesundheit. Traditionelle Organisations- und Regulierungsstrategien haben teilweise zur Informalisierung beigetragen. Der Prototyp des Gewerkschaftsmitglieds war und ist der Fabrikarbeiter, der abends zur Versammlung gehen und der streiken kann. Ohne diese Organisationsinstrumente verharmlosen zu wollen, ist zu konstatieren, daß die meisten Arbeiterinnen diese Handlungsoptionen nicht hatten/haben, weil sie abends Haushalt und Kinder zu versorgen haben und weil sie sich Lohnausfall durch Streik nicht leisten können. Arbeiterinnen wurden deshalb auch im entstehenden formellen Bereich als Schmutzkonkurrenz verteufelt.

„Überall betrachteten die Männer ihre weiblichen Arbeitsgenossen mit Haß und Mißtrauen und versuchten sich ihrer zu entledigen. ... Sie (die Arbeiterinnen) wurden zu Streikbrechern, ohne etwas anderes dabei zu empfinden, als Freude über die Arbeitsgelegenheit; sie ließen sich ausbeuten bis aufs äußerste und nahmen es hin, wie ein Faktum, wenn sie nur ihren Kindern dafür einen Tag lang den schlimmsten Hunger stillen konnten." (Lily Braun 1901: Die Frauenfrage, zit. nach GTB Düsseldorf 1981: 16)

Der geregelte und geschützte Arbeitsmarkt war schnell von männlichen Bewerbern beherrscht. Sie setzten die Normen. Nur wenn akuter Arbeitskräftemangel bestand oder wenn Männer zu teuer und zu wenig qualifiziert - im Sinne unbezahlter Qualifikationen - waren, wurden Frauen zugelassen. Zeitlich war die Familienarbeit bei einem Zehn- oder Zwölfstundentag ohnehin kaum zu meistern. Deshalb wurde Fabrikarbeit vorwiegend von jungen Frauen geleistet. Wie im vorigen Jahrhundert z.B. in die Bielefelder Leinenweberei kommen auch gegenwärtig oft junge, unverheiratete Frauen vom Lande in die heutigen asiatischen und zentralamerikanischen Exportzonen/Nähfabriken/Sweatshops ${ }^{3}$. Sie arbeiten, um mit Geldtransfers ihren Familien das Überleben zu sichern. Sie sind leichte Opfer der Deregulie-

3 „Sweatshop“ ist im nordamerikanischen Sprachgebrauch eine Produktionsstätte mit schlechten Arbeitsbedingungen. In Europa hat sich dagegen der Gebrauch als Synonym für kleine, informelle Produktionsstätten eingebürgert. „Maquila“ bezeichnet wiederum eine arbeitsteilig (tayloristisch) hochzergliederte, arbeitsintensive Fertigung meist für den Export in einer sogenannten Weltmarktfabrik. In der Bekleidungsindustrie geschieht das meist in Form des „outward processing trade“, „Cut-Make-and-Trim“, „Ishleme“, oder der „passiven Lohnveredelung“, bei der nur das arbeitsintensive, aber wenig wertschöpfende Nähen/Konfektionieren in der Weltmarktfabrik angesiedelt ist, alle anderen Produktionsstufen aber im Ausland bzw. extern. Weltmarktfabriken ihrerseits stehen oft in „Freien Produkionszonen“/,Exportzonen“ bzw. „Sonderwirtschaftszonen“. 
rung. Die Tatsache, daß es junge Frauen waren und sind, rechtfertigt a priori einen niedrigeren Lohn und die Außerkraftsetzung weiterer Regeln und Standards wie Mindestlöhne und Organisationsrecht. Die einzigen greifbaren Alternativen zur Näherei waren (vgl. GTB 1981: 38) und sind Hausangestelltentätigkeit oder Prostitution, also mindestens ebenso ungeregelte und ungeschützte Arbeiten.

Spezielle ,Schutz'-Gesetze behinderten parallel zur Ausbreitung der Fabrik den Zugang von Frauen zu Fabrikarbeitsplätzen (Hausen 1997: 723ff) ${ }^{4}$. Gegenwärtig fordern beispielsweise in Polen einige Frauenorganisationen den Abbau von Schutzgesetzen für Frauen. Ein übermäßiger Mutterschutz z.B. trägt dazu bei, geregelte Arbeitsplätze gegenüber Frauen protektionistisch abzuschotten. Dadurch wären viele Frauen gezwungen, in ungeregeltere Arbeitsverhältnisse abzuwandern (vgl. SOMO 1998, CCC 1998).

Gewerkschaften in Osteuropa z.B. scheuen vor Organisationsanstrengungen im Bekleidungsgewerbe zurück. Katholisch geprägte Gewerkschaften wie Solidarnosc in Polen weisen Arbeiterinnen die Hausfrauenrolle als angepaßter zu (CCC 1998, 13). Gerade in Osteuropa wird die Problematisierung der Diskriminierung von Frauen in der Lohnarbeit als nachrangig, unliberal, nicht marktkonform und ,retro-kommunistisch“ betrachtet (Dakova/Indshewa 1998; vgl. Jalusic 1997, 453ff). Dessen wollen sich GewerkschafterInnen natürlich nicht schuldig machen. Die herrschende Transformationskrise dient als Legitimation für diese Geringschätzung. Insgesamt wird die Lage von bezahlter und unbezahlter Frauenarbeit in Osteuropas Exportindustrie auch in der politischen Linken Westeuropas nicht hinreichend diskutiert. Die sozialwissenschaftliche Transitionsforschung ist geschlechtsblind (Jalusic 1997, 456).

Ende des 19. Jhs. gab es neben den genannten gewerkschaftlichen und sozialistischen Reformansätzen eine Frauenbewegung, die Heimarbeit thematisierte und Strategien entwarf. Eine Gruppe von Mittelschicht-Frauen versuchte, Heimnäherinnen in einer christlichen Gewerkschaft zu organisieren und Gesetze zum Schutz von Heimarbeit zu erreichen (Hausen 1986: 3ff). Diese Frauen wollten häusliche Lohnarbeit nicht zerstören, wie es das Ziel der Sozialisten war, sondern vielmehr ihre Lage durch Sozialversicherung, Lohnregelungen, Bildung und Berufsausbildung verbessern. Sie bewiesen damit wesentlich mehr Verständnis für die Lage der Heimnäherinnen. Die Sozialisten hatten es vorgezogen, die Frauen als hilflose Opfer zu betrachten. Freilich war das Fernziel dieser weiblichen Sozialreformer, das Ein-

4 Hausen weist nach, wie um die Jahrhundertwende die Sozialgesetzgebung zur Durchsetzung der bürgerlichen konventionellen Geschlechter-Rollen und -Regeln hinsichtlich der Arbeitsteilung in allen gesellschaftlichen Bereichen und zur Inthronisierung des männlichen proletarischen Familienernährers mit weiblicher Zuverdienerin/Hausfrau beitrug (Hausen 1997: 713 - 743). 
kommen des männlichen Familienernährers so weit zu erhöhen, daß dieser ,seinen Pflichten nachkommen' und seine Frau sich ganz der Familienarbeit widmen könne. In der Zwischenzeit sollte eine bessere Bezahlung die Vereinbarkeit von Familien- und Lohnarbeit erleichtern. Mindestlohn und branchenbezogene Lohnstandards spielten in der Diskussion um Regularien für die Heimarbeit eine entscheidende Rolle.

Nach wie vor sind es Frauenorganisationen wie Women Working Worldwide (Manchester) oder Committee for Asian Women (Hongkong), die die Situation von Frauen im informellen Sektor in das gesellschaftliche Bewußtsein bringen wollen und die Bezahlung in den Mittelpunkt ihrer Forderungen rücken. Die Kampagne für einen living wage - einen „existenzsichernden" Lohn, von dem ArbeiterInnen-Familien leben können ${ }^{5}$ - hat somit ihre Wurzeln nicht allein in Lohnkämpfen im formellen Sektor, sondern kommt vielmehr auch aus der Reformbewegung der Heimarbeit.

Schließlich gab es eine weitere Argumentationslinie aus akademisch untermauerter Arbeitgebersicht (vgl. Hausen 1986: 5ff), die noch der Vollständigkeit halber genannt werden soll. Sie ist insofern aktuell, als sie auch heute bei der Rechtfertigung extrem ausbeuterischer Formen informeller Arbeit wie der Prostitution gebraucht wird. Kontrastierend zu den Sozialisten wußten die Akademiker die freiwillige Entscheidung der HeimarbeiterInnen für diese Erwerbsform zu betonen, die nicht so armselig sei, wie von den Reformern dargestellt, zumal noch andere Einkommens- und Versorgungsquellen hinzukämen. Heimarbeit sei nicht die Ursache des Elends, sondern mache vielmehr aus der Not eine Tugend, indem strukturschwachen Regionen und eingeschränkten Arbeitskräften geholfen und eine weitere Migration in die Städte gemindert werde. ${ }^{6}$ Ziel dieser Argumentation ist es, jegliche regulierende Aktivität bezüglich des informellen Sektors zu delegitimieren.

Die verschiedenen Strategien und Argumentationslinien spiegelten sich im statistischen Erhebungsproblem um die Jahrhundertwende wieder: Waren HeimarbeiterInnen oder Selbstbeschäftigte nun Selbständige mit eigener Sozialversicherungs-Pflicht, oder waren sie abhängig beschäftigt von Auftraggebern, die die Sozialversicherungskosten zu tragen hätten (Komlosy 1997: 80)? Für die Gewerkschaften war und ist der Fall eindeutig: Da keine Tarifpartner existieren, kann es sich nicht um abhängig Beschäftigte han-

5 Im Unterschied zum Existenzminimum. Zur Definition eines living wage vgl. die ILOKonventionen Nr. 95 und 131. Innerhalb der living wage Kampagnen z.B. der Clean Clothes Campaign herrscht Konsens über die folgende Bestimmung eines living wage: Er ermöglicht es ArbeiterInnen, ihre Bedürfnisse nach ausgewogener Ernährung, sauberem Wasser, Unterkunft, Kleidung, Bildung, Gesundheitsversorgung, Beförderung zu befriedigen und beinhaltet darüber hinaus ein „discretionary income“.

6 Diese Argumentationslinie erinnert fatal an die in der Entwicklungszusammenarbeit übliche Begründung für einkommenschaffende Projekte für ländliche Frauen. 
deln. $\mathrm{Zu}$ fragen ist dennoch nach der wirtschaftlichen Abhängigkeit des informell Tätigen vom Auftraggeber - in der Regel ist sie sehr hoch.

Aktuelle Regulierungs-Versuche in Deutschland beinhalten eine Formalisierung der Scheinselbständigkeit und Bestrebungen zur Änderung des Betriebsverfassungsgesetzes. Es soll die Zugehörigkeit zum Unternehmen nicht mehr formal, sondern wirtschaftlich definieren. Ein Motiv für die Informalisierung durch globale Aus- und Verlagerung besteht darin, sich der wirtschaftlichen und sozialen Verantwortung für Lieferer bis hin zur Heimarbeiterin zu entziehen. Dies geschieht mit dem Verweis, sie seien formal selbständig und gehörten nicht zum Unternehmen ${ }^{7}$. Dabei handelt es sich um ein Standard-Argument, dessen sich Bekleidungshändler anfangs gegenüber der europäischen Clean Clothes Campaign bedienten, als die ersten Fälle gravierender Arbeitsrechtsverletzungen bei Zulieferern öffentlich gemacht wurden.

Die teilweise übermächtige wirtschaftliche und persönliche Abhängigkeit vom Auftraggeber illustriert ein aktuelles, fast unglaubliches Beispiel: Auf der Insel Saipan, einem US-amerikanischen Überseeterritorium, mußten chinesische Wanderarbeiterinnen unter schlechtesten Bedingungen arbeiten und für diese ,Arbeitsgelegenheit" eine ,Einstellungsgebühr' bezahlen, die durch die Arbeit abzuzahlen war - moderne Schuldknechtschaft. ${ }^{8}$

\section{Informelle Arbeit und osteuropäische Bekleidungsindustrie}

Das textile Verlagswesen und die Heimnäherei erlangten als passive Lohnveredelung ${ }^{9}$ Konjunktur in einem weiten globalen Spektrum von deregulierter Fabrikarbeit. Zu diesem Spektrum gehören Freie Exportzonen (Wick 1998) ebenso wie Heimnäherei. Die passive Lohnveredelung (PLV) beinhaltet traditionell tayloristisch organisierte Näharbeit in Niedriglohnlän-

7 Der US-Hemdenhersteller Philips Van Heusen begründete die Schließung einer eigenen Produktionsstätte in Guatemala (Camisas Modernas) damit, daß der harte Konkurrenzkampf flexiblere Zulieferstrukturen erfordere; Lieferbeziehungen zu eigenen Produktionsstätten seien zu schwerfällig - eine Rechtfertigung für Ex und Hopp/Cut and Run. Bei Camisas Modernas war gerade für Guatemala erstmals eine Gewerkschaft als Verhandlungspartner akzeptiert und ein Kollektivvertrag abgeschlossen worden. Gewerkschaften und NGOs sehen darin die eigentliche Begründung für die Fabrikschließung.

8 Ein Teil dieser Arbeiterinnen ist mit Unterstützung US-amerikanischer NGOs wie Global Exchange gegen die auftraggebenden US-Firmen, unter denen bekannte Markenartikler wie The GAP waren, vor Gericht gezogen und hat einen Vergleich erzielt, der die Rückzahlung dieser Gebühren und das erste unabhängige Monitoring einschließt. Die Möglichkeit eines US-Prozesses ergab sich daraus, daß die Bekleidung als „Made in USA“ verkauft wurde, da Saipan zu den USA gehört.

9 Passive Lohnveredelung (PLV) - auch „Outward Processing Trade/OPT“, „Lohnsystem“, „Ishleme“ genannt - bedeutet, daß wie im Verlagswesen die Ausgangsmaterialien, also meist die fertig zugeschnittenen Kleiderteile einschließlich der Accessoires zugeliefert, am Fabrikationsort nur zusammengenäht und verpackt werden, um dann wieder reexportiert an den Auftraggeber (Verleger) zurückzugehen. 
dern. Auch dort kann wieder mittels deregulierter, unterbezahlter Lohnarbeit und unbezahlter Subsistenzarbeit eine Arbeitsrente angeeignet werden. Das gilt insbesondere für die Haupt-Herkunftsregion lohnveredelter Bekleidung Westeuropas: Osteuropa.

Unter den zehn größten PLV-Bekleidungs-Lieferländern für die BRD waren 1998 nur drei nicht-osteuropäische Länder: Tunesien (3.Stelle), Marokko (8.) und die Türkei (10.) (Markert 1999a). Die Textil- und Bekleidungsindustrie $^{10}$ weist „eine - für den sonstigen Handel atypische - rasch und überdurchschnittlich zunehmende Handelsverflechtung zwischen Ost und West" auf (Gälli 1992, 12).

Folgende Gründe sprechen für eine Verlagerung der Näherarbeit nach Osteuropa:

1. Die Nähe zum Markt.

2. Dass die Arbeitskräfte sowie die technologischen und infrastrukturellen Voraussetzungen vorhanden sind, sowie Liefertreue.

3. Währungsabwertung und Austauschrelationen: Alle osteuropäischen Währungen wurden abgewertet. Bulgarien rangiert gemessen am Dollaräquivalent der Löhne von 1996 an letzter Stelle unter den Transformationsökonomien (UNDP 1998, 43). Die Abwertung garantiert sehr günstige Austauschrelationen für westeuropäische und nordamerikanische Auftraggeber - auf Kosten der inländischen Kaufkraft dieser Löhne, denn importierte Produkte werden deshalb nicht billiger und Inflation kommt hinzu ein Kreislauf, wie er aus „Entwicklungsländern“ bekannt ist.

4. Privilegierter Zugang Osteuropas zum EU-Markt durch Aufnahme in die EU bzw. EU-Assoziierung: Die Handelspolitik der EU begünstigt den passiven Lohnveredelungsverkehr mit Osteuropa. Deshalb ist der Anteil der PLV-Ausfuhren am Gesamtexport von Bekleidung dieser Länder relativ hoch (ca. 80\%), dagegen in der Türkei relativ niedrig (ca. 15\%). EUMitgliedschaft bzw. -Assoziierung sichert zunächst zoll- und quotenfreien Zugang zum EU-Markt (ohne Einfuhrgenehmigungspflicht ${ }^{11}$ ) und unterwirft die Länder gleichzeitig dem Druck der Anpassung an EU-Kriterien und -Normen. Dies führt beschleunigt zur Rücknahme sozialer Sicherungen. ,Übertriebene' Sozialgarantien sind für eine marktwirtschaftliche Transformation nicht förderlich. Die mit der EU-Osterweiterung verbundene handelspolitische Strategie gibt Osteuropa einen Sonderstatus gegenüber den sogenannten Entwicklungsländern und bietet somit eine gute Grundla-

10 Die Bekleidungsindustrie umfaßt die Konfektionierung, das Fertigen von Kleidung für den Massenbedarf, für den Kauf „von der Stange“. Die Textilindustrie produziert die Stoffe und textilen Flächen dafür wie auch für andere Branchen wie die Autoindustrie.

11 Quoten- und Einfuhrgenehmigungsfreiheit sowie Befreiung von der Bewilligungspflicht für den „Lohnveredelungsverkehr“ gilt allerdings nur für Polen, Ungarn, Bulgarien, Rumänien, die Tschechische und Slowakische Republik - die EU teilt und herrscht. 
ge für die Ausrichtung der Volkswirtschaften und Gesellschaften Osteuropas an den Handels- und Investitions-Bedürfnissen der EU bzw. Westeuropas.

5. Positives Image von westlichem wirtschaftlichem Engagement: Während Anti-Sweatshop-Bewegung in Nordamerika und Clean Clothes Campaign (CCC) in Westeuropa die Bekleidungsproduktion in Asien und Mittelamerika anrüchig gemacht haben, erscheint die ,Aufbauhilfe' in Osteuropa in der Öffentlichkeit als ,reform-fördernd“. Die soziale Dimension der Transformation Osteuropas ist bisher meist einseitig als SozialdumpingGefahr für den Westen (u.a. bei Huber 1999, 38) dargestellt worden. Diese Darstellung verhinderte weitgehend eine Beschäftigung mit den riesigen negativen sozialen Folgen der Transformation Osteuropas. ${ }^{12}$ Auch bei der Ost-Erweiterung der EU spielen soziale Aspekte praktisch keine Rolle ${ }^{13}$ (siehe Langewiesche 1998). Dazu trägt bei, daß soziale Desintegrationstendenzen in Westeuropa selbst ebenfalls nicht angemessen thematisiert werden (Potel 1999, Langewiesche 1998, 311, 324).

All dies hat zur Maquiladorisierung Osteuropas geführt: Mit WendeMarktöffnung und EU-Osterweiterung erfolgt die Zurichtung der osteuropäischen Wirtschaften zum wohlfeilen Hinterhof Westeuropas. Ähnlich wie in Ländern Mittelamerikas war die vormals binnenmarktorientierte Wirtschaft und Bekleidungsindustrie durch die marktwirtschaftliche Transformation zusammengebrochen. Das mit der EU-Integration häufig verbundene Versprechen einer nachholenden Entwicklung bleibt ebenso wie in Mittelamerika eine gefährliche Illusion. Osteuropas Industriereste überleben als arbeitsintensive bzw. rohstoffliefernde Hilfsindustrie. Sie , spezialisieren" sich auf einzelne Glieder einer ansonsten technologisch differenzierten Fertigungskette, von der sie weitgehend abgekoppelt sind. Bekleidungsproduzenten wie die Firma Steilmann und KfW-(also steuer-) finanzierte Beratungsbüros unternehmen einige Anstrengungen in Osteuropa, um Management und Personal zu qualifizieren sowie die innerbetriebliche Arbeitsorganisation zu verbessern (vgl. Steilmann 1999, Biskupek 1998). Damit festigen sie die Spezialisierung auf sehr fragile Wirtschafts-Nischen. Die in dem Zusammenhang gar „entwicklungspolitisch“ vorgebrachte Forderung nach „Qualifizierungsoffensiven“ für Osteuropa ignoriert sowohl die konkreten Bedingungen wie auch die dargelegten strukturellen Zusammenhänge.

Bei dieser Forderung, so gerechtfertigt sie im einzelnen ist, bleibt zudem jeder Bezug zum Lohn unberücksichtigt, was sie einseitig und unglaubwürdig macht. Dahinter steht ein Konzept, das schlechte Arbeitsbedingun-

12 Im Gegensatz zu ökologischen Problemen.

13 Auch das Europäische Gewerkschaftsinstitut hat sich erst in seinem 97er Jahrbuch mit der sozialen Dimension der EU-Ost-Erweiterung beschäftigt. 
gen zum einen aus Defiziten des Arbeitskraftangebots (mangelnde Qualifikation) und/oder der geringen Nachfrage im formellen Sektor erklärt (ILO 1998: 21). Die ILO z.B. empfiehlt Frauen folgerichtig, mit Bildungsmaßnahmen und Unternehmensgründungen ihre Situation zu verbessern.

Im ehemaligen RGW gab es im Unterschied zu Mittelamerika eine wesentlich entwickeltere industrielle Basis und einen hohen Bildungsstand der Bevölkerung. Die gesamte textile Kette der Mitgliedsländer war arbeitsteilig organisiert. Diese Kette mußte mit der Marktöffnung und der Umstellung auf die DM reißen (vgl. Bast-Haider 1997: 93ff). Die Art, wie der RGW den Textil-/Bekleidungssektor organisiert hatte, war für die kapitalistische Organisation des Sektors untauglich. Die Konkurrenz wird auf vorund ausgelagerte Stufen der textilen Kette angeheizt („Leanterror"). Handelspolitische und wirtschaftspolitische Regeln stimulieren die Bildung vieler Zulieferbetriebe für westeuropäische oder nordamerikanische Auftraggeber (Ansiedlungsförderung damals durch Aufteilung von Almenden, heute z.B. durch Ausweis von Freien Exportzonen). Das Verlags-Muster viele, dezentrale Fertigungsstätten für eine Design- und Managementzentrale -, rationalisierte die globale Bekleidungsindustrie. Es bedeutet verschärfte Konkurrenz und vermehrtes Ausspielen der Zulieferländer, der Zulieferer und letztlich der ArbeiterInnen gegeneinander. Ein so angeheizter globaler Konkurrenzkampf funktioniert nur mit viel Ideologie und Mythen wie dem des Wohlstandsversprechens bei EU-Mitgliedschaft.

Im 19. Jh. wurde die unterbezahlte, ungeregelte Arbeit weiter an die globale Peripherie gedrängt und gleichzeitig die strukturschwachen Regionen Westeuropas selbst deindustrialisiert (vgl. Komlosy 1997: 83). Das ländliche Gewerbe dort war nicht mehr konkurrenzfähig. Als Folge marktwirtschaftlicher Transformation in Osteuropa vollzieht sich gegenwärtig eine ähnliche, jedoch wesentlich schnellere Deindustrialisierung. Auch dieser Übergang zum Kapitalismus erfordert die Außerkraftsetzung bisheriger Regelwerke eines durchregulierenden Staates. Die Regeln, die in Osteuropa aufgehoben wurden, sind Sozialgarantien (konservierter Fordismus), die die Transformation nur behindern.

Da diese Außerkraftsetzung vorkapitalistischer Regeln ${ }^{14}$ von neoliberalen Dogmen geleitet ist und mit einer globalisierten Deregulierungspolitik zusammenfällt, bilden die Überreste des Wirtschaftens kaum mehr als Varianten unterbezahlter, ungeschützter, praktisch unregulierter Arbeit.

14 Die Rolle der Privatisierung in diesem Zusammenhang kann hier aus Platzgründen nicht behandelt werden. 


\section{Arbeits- und Lebensbedingungen in der ,Nähstube“ Europas: ,wel- come to the South" (Dan Gallin) ${ }^{15}$}

Die Produktion im „Lohnsystem“ ist zunächst für viele Betriebe (staatliche wie neue private) die einzige Überlebenschance, obgleich gegenüber dem „Vollgeschäft“ wenig profitabel. „Lohnsystem“-Fertigung ist unsicher und dem Auftraggeber ausgeliefert - sie ist ,flexibel'. Die Schwankungen und Unsicherheiten seines Geschäfts kann der Auftraggeber einfacher auf den Zulieferer abwälzen. Handelskonzerne bevorzugen die PLV in Osteuropa für „rush orders“, schnelle Aufträge. Immer kürzere Auftragsabwicklungszeiten werden abgepreßt.

Auch bei der Maquiladorisierung Osteuropas spielt das weibliche Arbeitsvermögen und seine Unterbezahlung eine entscheidende Rolle. Es sind zu 80 bis $90 \%$ Frauen, die in der Konfektion arbeiten. Die „Fertigkeit der Polinnen“ (Gälli 1992, 19) z.B. dient als Geschlechterstereotyp zur Legitimation der Bevorzugung junger Frauen verbunden mit einem ZuverdienerinLohn. In der Regel wird nicht existenzsichernder Lohn gezahlt. Die Löhne im Bekleidungsgewerbe gehören weltweit auch in Deutschland, zu den niedrigsten im Branchen-Vergleich. Zwischen Juli und September 1997 lag der Durchschnittslohn pro Monat in der bulgarischen Bekleidungsindustrie bei 108 DM. Gesamtwirtschaftlich wurden durchschnittlich 158 DM gezahlt (Berechnungen des sozialstatistischen Büros ISTUR der Gewerkschaftskonförderation CITUB ${ }^{16}$ ). In der polnischen Bekleidungsindustrie besteht ein ähnliches Verhältnis zwischen dem gesamtwirtschaftlichen und dem Durchschnittslohn in der Bekleidungsindustrie (CCC 1998, 10). Für Oktober 1998 wurde in Bulgarien ein Basisminimaleinkommen von 32,40 DM und ein Mindestlohn von 53,50 DM (in Rumänien zur selben Zeit: ca. 120 DM) festgelegt. Dem niedrigen Lohnniveau stehen Lebenshaltungskosten von 840 DM pro Monat für einen durchschnittlichen 4-PersonenHaushalt gegenüber (im selben Zeitraum Juli bis September 1997 - Nationales Statistik-Institut NSI und ISTUR). Nicht wenige Statistiken belegen die wachsende Verarmung vieler Menschen in Osteuropa (z.B. UNDP 1998, 43/44), auch wenn sie Arbeit haben. Bezahlte Arbeit allein sichert für die Mehrheit nicht das Überleben - sie sind „,working poor“. Überleben können die Familien nur durch Wochenendeinsätze in der Subsistenzwirtschaft der Großmutter oder des Onkels auf dem Dorfe, von der sie sich miternähren. Die Migrationsbewegungen haben sich umgekehrt zugunsten des Landes (UNDP 1998, 64). Das Subsistenz-Großfamilien-Modell dominiert in Osteuropa gegenüber dem Transfer-Modell Asiens oder Mittelamerikas, wo junge Frauen vom Lande in die Sonderwirtschaftszonen migrieren und

15 Auf der Konferenz „Entwicklungspolitische NROs und EU-Erweiterung“Wien 1998.

16 Confederation of Independent Trade Unions of Bulgaria, bulg.: KNSB 
ihren Familien Teile ihres eigenen Lohnes unter großen persönlichen Opfern transferieren.

Langfristige Lebensstrategien weichen kurzfristigem Überleben. OsteuropäerInnen und insbesondere Frauen können es sich in der Regel nicht leisten, über die eigene Qualifikation und die ihrer Kinder nachzudenken, sondern müssen ihre gesamte Energie dem täglichen Überlebenskampf widmen. Auch in Osteuropa nimmt die soziale Desintegration infolge der marktwirtschaftlichen Transformation und Verarmung zu. Gleichzeitig reproduzieren sich großfamiliäre Strukturen in der Subsistenzwirtschaft, aber auch im „Klientelismus“. Für Informalisierungs-Tendenzen ist ein solcher struktureller Dualismus charakteristisch.

Die Zurichtung der Volkswirtschaften auf „Lohnveredelungsverkehr“ und die entsprechende Ausbeutung des weiblichen Arbeitsvermögens zeigt sich exemplarisch in drei typischen Bereichen der „Lohn“-Näherei Bulgariens, die sich ähnlich auch in anderen osteuropäischen Ländern wiederfinden (CCC 1998): Erstens in der ,reinen' Schattenwirtschaft, zweitens im ehemaligen oder noch staatlichen Sektor und drittens in neuen privaten Firmen vorzugsweise in Regionen mit hoher Arbeitslosigkeit.

\section{Aufschwung der Schattenwirtschaft}

Schätzungen der bulgarischen Gewerkschaftskonföderation Podkrepa zufolge beträgt der Anteil der Schattenwirtschaft am BIP (Bruttoinlandprodukt) ca. 50\% (Lozanov 1998). Sie hat den „freien Fall“ (Kerstin BastHaider) der Bekleidungsindustrie etwas gemindert. Speziell an der griechischen Grenze schießen viele Garagennähstuben wie Pilze aus dem Boden, werden „abgeerntet“, wieder geschlossen und entstehen woanders neu. (Das Prinzip ähnelt dem der mexikanischen Maquilas an der Grenze zu den USA.) Für griechische Zulieferfirmen ist die Region attraktiv, da sie für die Unterauftragsvergabe nur die Hälfte bis zu einem Drittel des Lohnes kalkulieren müssen. In dieser Region leben Minderheiten wie die islamischtürkische. Vertreterinnen dieser Minderheit sind als fleißige und billige Arbeitskräfte begehrt. Der dramatische Rückgang bei den Schuleinschreibungen von Mädchen wird der Tatsache zugeschrieben, daß viele Mädchen dieser Minderheit aufhören oder nie beginnen, die Schule zu besuchen (UNDP 1998, 52, Dakova/Indshewa 1998). Sie werden wieder auf alte Geschlechterrollen festgelegt. Geschlechtsspezifische und rassistische $\mathrm{Zu}-$ schreibungen verflechten sich. Auch in den Sweatshops von Los Angeles werden untragbare Arbeitsbedingungen so legitimiert (Bonacich 1997: 144ff). Sie begründen ideologisch die Arbeitsmarkt-Segregation und Lohnkonkurrenz zwischen den Geschlechtern und den Ethnien.

17 In vielen Teilen Rußlands bleibt der Naturalaustausch nicht auf die Subsistenzwirtschaft beschränkt, sondern ersetzt fast vollständig das Geld (Meyer 1998). 


\section{Staatlicher Sektor und Privatisierung}

Der staatliche Sektor, einst ausgewiesen durch vollständige soziale und Beschäftigungs-Sicherheit, ist zu einem Instrument der beschleunigten Ableitung gesellschaftlicher Ressourcen und der Dekapitalisierung der Industrie mutiert (UNDP 1998, 26). Das einstige Vorzeigestück sozialer Sicherung wird massiv informalisiert. Die Beschäftigten sind nach ihrer Dezimierung (auf ca. 30\% in einem Beispiel) und nach jahrelanger Abwicklung/Privatisierung eingeschüchtert und ohne Hoffnung. Gewerkschaftliche Vertrauensleute wurden zum Teil gesetzeswidrig entlassen. Ehemals oder noch staatliche Betriebe sind die fast einzigen Produktionsstätten, in denen es noch eine nennenswerte gewerkschaftliche Organisation gibt. ${ }^{18}$ Sie existiert allerdings nur als Mitgliederzahl auf dem Papier, real haben Gewerkschaften kaum Einfluß.

In der Regel sind Beschäftigungssicherheit und Lohn in privaten Firmen höher als in staatlichen Betrieben. Die unsichere Situation staatlicher Unternehmen läßt die formal den Gesetzen entsprechenden Arbeitsverträge zu wertlosem Papier werden. Die Regelungen in formellen Arbeitsverträgen staatlicher Betriebe sind folgenlos. Der staatliche Sektor in der Bekleidungsindustrie bietet praktisch noch unsicherere und ungeregeltere Beschäftigung als halbformelle private Firmen oder selbst als die Schattenwirtschaft.

\section{Private Nähereien in Gebieten mit hoher Arbeitslosigkeit}

Die Arbeitslosigkeit nimmt durch Entlassungen im staatlichen Sektor zu, die durch den privaten Bereich nicht annähernd aufgefangen werden können. Anfang der 90er Jahre, also sofort nach der „Wende“, ist die Arbeitslosigkeit unter Frauen sehr schnell angestiegen. Die Arbeitslosenraten differieren stark zwischen einzelnen Regionen, und diese Differenzierung nimmt zu (für Bulgarien: UNDP 1998, 46). In einigen Kreisen erreicht sie (offiziell) $30 \%$. Viele nicht Arbeitende registrieren sich jedoch nicht als „arbeitslos“, da es als entwürdigend und zwecklos gilt.

Regionen mit hoher Arbeitslosigkeit werden von Konfektionären bevorzugt bzw. dort bleiben Konfektionsbetriebe eher bestehen. Es eröffnen sich vielfältige Möglichkeiten, Gesetze zu umgehen oder Druck auf ArbeiterInnen auszuüben, weil sie weniger Lohnarbeits-Alternativen haben. Z.B. werden NäherInnen in privaten Firmen nur für den staatlich festgelegten Mindestlohn eingestellt, den Rest erhalten sie bar ohne Sozialabgaben. Selten gibt es einen Arbeitsvertrag. Als Einstellungsvoraussetzung werden Frauen aufgefordert, sich nicht gewerkschaftlich zu engagieren. Frauen, insbesondere jungen Frauen, werden häufiger Jobs auf Zeit, zum Minimallohn und ohne

18 Ausführlicher zum Problem der Interessenvertretung und gewerkschaftlicher Politik vgl. Musiolek (1999) 
Sozialversicherung angeboten als Männern, und Frauen nehmen diese Angebote gerade in strukturschwachen Regionen öfter an (Dakova/Indshewa 1998). Im privaten Bereich wird nach allgemeiner Einschätzung das Arbeitsgesetz mindestens teilweise ignoriert. Das betrifft besonders Frauen, denn gerade sie sind auf ein schützendes Arbeitsrecht angewiesen. Die Praktiken in osteuropäischen Betrieben nähern sich schnell denen in asiatischen und mittelamerikanischen Unternehmen an: Kinderarbeit, unbezahlte Überstunden, sexueller Mißbrauch etc. (Dakova/Indshewa 1998 sowie bulgarische Zeitungsberichte) sind keine Seltenheiten mehr. Arbeitsinspekteure werden persönlich bedroht. Da sie zudem völlig überfordert sind, können und sollen sie nicht genau kontrollieren. Ihre Strafen gegenüber Firmen werden den ArbeiterInnen zuweilen noch vom Lohn abgezogen. In strukturschwachen Regionen arbeiten Frauen in Bekleidungsbetrieben zu besonders schlechten Konditionen, während ihre Männer arbeitslos sind, was nicht selten zu familiären Konflikten führt.

Dieser private Bereich überschneidet sich häufig mit dem erst- und zweitgenannten: Private Betriebe gingen aus staatlichen Unternehmen hervor, indem z.B. der Direktor seine eigene Firma aufmachte und das gewünschte Personal abwarb, oder die Näherei ist aus der reinen Schattenwirtschaft herausgewachsen. Neben dem eigentlichen informellen Sektor gibt es also einen großen Sektor schleichender Informalisierung - Neoinformalität. ${ }^{1}$

Welches Beispiel könnte die Situation in diesem Bereich der Bekleidungsherstellung besser illustrieren als die Lohnnähereien südkoreanischer Produzenten im russischen Vladivostok mit chinesischen und russischen Arbeiterinnen? Kommentar eines Regionaladministrators: „Es gibt nicht viel Gutes, was man über das Arrangement sagen kann, aber die Arbeitslosigkeit ist einfach zu hoch. Zumindest bezahlen sie regelmäßig den Lohn. ${ }^{20}$

\section{Zivilgesellschaftliche Initiativen für die Einbeziehung informalisierter Näharbeit in soziale Regulierungsansätze}

In internationalen zivilgesellschaftlichen Bewegungen gibt es zwei Ansätze, die hier kurz vorgestellt werden sollen: Die Einbeziehung der gesamten Beschaffungskette für Bekleidung einschließlich informell arbeitender Näherinnen erstens in den Geltungsbereich von „Corporate Codes“ (Verhaltenskodices) und zweitens in die Forderung nach einem ,living wage“.

19 Wenn z.B. der OTTO-Versand ihm entfernt zuzuordnende Call Center in Mecklenburg unterhält, so wird dort kein im HBV-Bereich üblicher Tariflohn gezahlt und gewerkschaftliche Organisation ist in Anbetracht der hohen Arbeitslosigkeit dort ein Fremdwort.

20 „Russia's patchwork economy: South Korean companies, Chinese workers and U.S. Entree" New York Times, March 18th. 


\section{Verhaltenskodices}

Zivilgesellschaftliche Bewegungen haben sich dem Instrument der Verhaltenskodices zugewendet. Der entscheidende Grund dafür war, die realen, wirtschaftlich begründeten Verantwortungen entlang den unübersichtlichen globalen Beschaffungsketten auszumachen und einzufordern. Dies sollte unabhängig davon geschehen, wer der unmittelbare Arbeitgeber ist. Informell Beschäftigte wurden in die Definition von „ArbeitnehmerInnen“ im Kodex der Clean Clothes Campaign (CCC) einbezogen ${ }^{21}$. Besonders Frauenorganisationen und Selbstorganisationen von ArbeiterInnen haben auf dieser Ausgestaltung des Geltungsbereiches bestanden. Es war aber auch ein Ergebnis des Diskurses über Sozialklauseln in Handelsverträgen. Viele Organisationen, die der CCC angeschlossen sind, kritisierten an den Sozialklauseln die fehlende Einbeziehung der Verantwortung der Modemultis gegenüber allen für sie Arbeitenden und den potentiellen Protektionismus. Beides würde die letzten Glieder der Beschaffungskette, also Heim- und Sweatshop-ArbeiterInnen, bestrafen. So kritisiert die CCC am Modell Social Accountability (SA) $8000^{22}$ der US-Organisation Council on Economic Priorities vor allem, daß die Verantwortung der Händler und großen Produzenten nicht klar definiert wird. Diese können ihre Verantwortung an die Zulieferer delegieren, indem sie von diesen SA 8000-Zertifizierung verlangen. Das Hongkonger Netzwerk Labour Rights in China (LARIC) stellt fest, daß wie viele andere Verhaltenskodices SA 8000 informell Arbeitende nicht einschließt (LARIC 1999: 2).

Bislang hat nur der schweizerische Handelskonzern Migros diesen Geltungsbereich für seinen Corporate Code mit einem Bekenntnis zum gesamten Arbeitsverhaltenskodex der CCC übernommen ${ }^{23}$. Andere wie z.B. C\&A erwähnen ,third parties“ und Subunternehmer eher mit der Konnotation, ungeliebte Unterauftragsvergabe auszuschalten.

Die Internationale Arbeitsorganisation ILO beschäftigt sich in ihrer Bilanz verschiedener ,privater Initiativen zu Arbeits-Themen wie Verhaltenskodices" nicht mit diesem Problem des Geltungsbereichs (ILO 1998). 1996 hat sie eine Konvention zur Heimarbeit verabschiedet, die sie in der genannten Bilanz jedoch nicht erwähnt. Die jahrelangen ergebnislosen Diskussionen in der ILO über die Verabschiedung einer Konvention zur Vertragsarbeit

$21 \mathrm{Zu}$ Entstehung und Wirkung des Instruments Verhaltenskodex und zum Wortlaut des zitierten Kodex vgl. Musiolek (1999).

22 Ein an das ISO-Vorgehen angelehntes Zertifizierungs-Modell, das ähnliche Standards wie der CCC-Kodex enthält, allerdings in den Durchführungsbestimmungen (guidence documents) wichtige Fragen offen läßt.

23 Vgl. Migros Code of Conduct for the socially acceptable production of clothing and shoes - Migros ist das erste Unternehmen, das den Arbeitsverhaltenskodex der CCC einschließlich der Monitoring-Bestimmungen durch einen Letter of Intent kürzlich übernommen hat. 
zeigen ihr grundsätzliches Dilemma: Die traditionellen sozialen, korporatistischen Strukturen, die der dreigliedrigen ILO-Struktur zugrunde liegen, werden zunehmend ausgehöhlt u.a. durch die Informalisierung.

Die FairWear-Bewegung in Australien hat einen speziellen HeimarbeiterInnen-Kodex (Homeworkers Code of Practice) ausgeschrieben, der vor allem einen living wage und die Gewährung des Organisationsrechts fordert. Diese Initiativen tragen der Tatsache Rechnung, daß es in Australien viele HeimnäherInnen gibt. Auch die kanadische Anti-Sweatshop-Bewegung, insbesondere das Maquila Solidarity Network, setzt bei der Sichtbarmachung der eigenen Heim- und Vertragsarbeitsbedingungen an. Das Netzwerk fordert in erster Linie eine gemeinsame rechtliche Verantwortung der Händler und Produzenten einschließlich des unmittelbaren Arbeitgebers für die Verletzung von Arbeitsstandards ihrer Auftrags- und Unterauftragsnehmer (Yanz/Jeffcott et al. 1999: 23).

\section{Living wage}

Der Forderung nach einem living wage wird von den meisten Organisationen in ProduzentInnen-Ländern auch in Osteuropa höchste Priorität gegeben. Wie die Genese dieser Forderung zeigt, wird sie nicht zuletzt deshalb erhoben, um die Arbeitsbedingungen im informellen Sektor zu verbessern. Trotzdem hat sich erst vor kurzem eine internationale Kampagne für die Durchsetzung eines living wage formiert. Die Angst um den Verlust des Arbeitsplatzes ließ viele Organisationen zurückschrecken. Entsprechende Kampagnen starteten 1998 in den USA u.a. durch die NGOs Global Exchange und Sweatshop Watch und 1999 in Großbritannien durch das Labour behind the Label-Netzwerk und die Ethical Trading Initiative (ETI). Auch der CCC-Arbeitsverhaltenskodex enthält die Zahlung eines living wage. Explizit wird in diesen Initiativen auf den informellen Sektor Bezug genommen.

Doch nur ungefähr jeder zweite Firmenkodex enthält gegenwärtig einen Passus über Löhne. Meist wird darin auf nationale Mindestlöhne Bezug genommen (vgl. ILO 1998: 16) - so auch von der US-Apparel Industry Partnership (AIP) ${ }^{24}$, einer Regierungsinitiative zur Bekämpfung der Sweatshops. Wie das osteuropäische Beispiel zeigt, liegen diese Mindestlöhne in der Regel jedoch weit unter dem Niveau eines existenzsichernden Lohns und in nicht wenigen Fällen sogar unter der Weltbank-Armutsgrenze von einem US-Dollar pro Tag. Im übrigen werden selbst diese Mindestlöhne in unregulierten Arbeitsbereichen nicht unbedingt eingehalten.

24 Zur AIP und nordamerikanischen Initiativen vgl. Artikel von Edna Bonacic/Jill Esbenshade, Ronald Köpke und Christian Mücke in: Musiolek (1999). 
Das kanadische Maquila Solidarity Network fordert eine Registrierung für HeimarbeiterInnen und einen Branchenkollektivvertrag für alle ArbeiterInnen einschließlich der HeimarbeiterInnen (Yanz/Jeffkott et al. 1999: 23ff). Damit knüpft es direkt an Forderungen aus der Debatte um die HausarbeitsReform der Jahrhundertwende an (vgl. Hausen 1986: 4), bricht aber auch mit einem Tabu der Gewerkschaftsbewegung. Mit einem Tabu brach ebenfalls eine Aktion australischer Gewerkschafter aus der Bergbau- und Baubranche, die im Sommer vor Markenläden einer Shopping Mall Sydneys gegen die Unterbezahlung von Heimnäherinnen protestierten, indem sie sich bis auf das legale Maß auszogen.

\section{Resümee}

Die flexibel abrufbare Teilzeitarbeit in der Heimindustrie subventionierte die Verlags-Großbetriebe in den Städten, den Zentren des entstehenden Kapitalismus. Heute subventioniert die unterbezahlte, deregulierte Fabrikund Hausarbeit osteuropäischer NäherInnen ,Weltkonzerne' wie Adidas, H\&M oder C\&A. Die kapitalistische Entwicklung hat den informellen Sektor keineswegs automatisch verdrängt. Sie hat im Gegenteil immer wieder ungeregelte sowie un- und unterbezahlte Arbeit hervorgebracht. Durch die Abwesenheit von Regeln im informellen Bereich wurde und wird Druck auf Lohn und Arbeitsintensität in den geregelten Segmenten ausgeübt. Insofern ist der informelle Sektor eine kontinuierliche Bedingung kapitalistischer Rationalität. Verdrängt wurde die informelle Arbeit allerdings in der Tat aus dem öffentlichen Bewußtsein.

Osteuropa als , verlängerte Werkbank' Westeuropas wird praktisch in Gänze dereguliert. Und das geschieht in einem Tempo, das Problematisierungen der sozialen Auswirkungen schon zeitlich kaum zuläßt. Gleichzeitig werden die Menschen mit Mythen einer nachholenden Entwicklung abgespeist, die nicht zuletzt von der EU-Administration immer wieder genährt werden.

Die Geschichte sozialreformerischer Bestrebungen hinsichtlich des informellen Sektors verdeutlicht ein Dilemma dieses Diskurses: die Geschlechterblindheit bzw. die bewußte Verdrängung von Frauen durch einen Teil dieser Sozialreformer, speziell vieler Gewerkschaften. Die historische Entwicklung der Koexistenz von formeller und informeller Arbeit im Textilund Bekleidungsgewerbe ist eine Geschichte der Verflechtung von sozialer und Geschlechterfrage. Die Debatte um die Reform der Heimarbeit war letztlich ein Verteilungskampf zwischen formell und informell Arbeitenden auch mit Hilfe von Geschlechterstereotypen. Eine Einordnung informalisierter Tätigkeiten in Kategorien gestaltet sich vor allem deshalb schwierig, weil das dominierende Kategoriengebäude von der scheinbaren Regel aus- 
geht - von geregelten Arbeitsverhältnissen, vom fordistischen Industriearbeitsplatz einschließlich des vorwiegend männlichen ,Normalarbeitsverhältnisses'.

Dieses Dilemma hat die rigorose Informalisierung von arbeitsintensiven Zuliefertätigkeiten in Osteuropa beschleunigt. Es nimmt nicht Wunder, daß sich gegenwärtig besonders zivilgesellschaftliche Bewegungen, die mit einigen Gewerkschaften kooperieren, der Einbeziehung informalisierter (Frauen-)Arbeit in Regulierungsansätze widmen.

\section{Literatur}

Altenburg, Tilman/Walker, Ian (1995): Nationale und regionale Entwicklungsimpulse durch Weltmarktfabriken. Beispiele aus Zentralamerika, in: Zeitschrift für Wirtschaftsgeografie, Jg. 39, Heft 2, 102-112

Ascoly, Nina/ Zeldenrust, Ineke (1998): Codes of Conduct for Transnational Corporations. An Overview. IRENE. Tilburg, The Netherlands

Arbeitsgruppe Alternative Wirtschaftspolitik (1997): Memorandum europäischer Wirtschaftswissenschaftler und Wirtschaftswissenschaftlerinnen: „Vollbeschäftigung, sozialer Zusammenhalt und Gerechtigkeit für Europa - Alternativen zum Austeritätswettlauf" Zirkular Nr. 25 Bremen

Bast-Haider, Kerstin (1997): Branchensterben - Frauen aus der ostdeutschen Bekleidungsindustrie, in: Ich bin chic, und du mußt schuften: Frauenarbeit für den globalen Modemarkt Bettina Musiolek (Hrsg.), Frankfurt a.M., 93-101

Bickham Mendez, Jennifer/Köpke, Ronald (1999): Gender und Globalisierung: Zentralamerikanische Frauenorganisationen zwischen Transnationaler Konkurrenz und Kooperation in der Bekleidungsindustrie, in: Peripherie. Soest, 75/ 1999

Biskupek, Thomas (1998): Ein Stück Zukunft im Osten, in: BW 20 1998, 3 - 15

Bonacic, Edna (1998): The Labor behind the Label - wie die „ModemacherInnen“ in Los Angeles/USA arbeiten, in: Ich bin chic, und du mußt schuften: Frauenarbeit für den globalen Modemarkt Bettina Musiolek (Hrsg.), Frankfurt a.M., 144-157

Clean Clothes Campaign (1998): Made in Eastern Europe, Amsterdam

Clean Clothes Campaign (Hrsg. 1999): A code for homeworkers: Australia's Fair Wear Campaign, in: Clean Clothes Newsletter, Amsterdam, no. 11 August, 9

Da Bindu Collective (1998): News Paper Free - Trade - Zone, Ja-Ela/ Sri Lanka Dec.

Dakova, Vera B./Indshewa, Regina (1999): Bulgaria - Social Rights in Transition - Gains and Losses, Manuskript, Sofia

Dannecker, Petra (1998): Globalisierung und „Migration” von Produktions- und Geschlechterstrukturen: Fabrikarbeiterinnen in Bangladesh. Bielefeld

David, Natacha, Internationaler Bund Freier Gewerkschaften/ IBFG (Hrsg. 1996): Zwei Welten: Frauen und die Weltwirtschaft, Brüssel

DGB Bildungswerk, Nord-Süd-Netz (Hrsg. 1995): Informeller Sektor - Markwirtschaft im Schatten, Düsseldorf

Gälli, Anton (1992): Nöte und Perspektiven der Textil- und Bekleidungsindustrie in Ostmitteleuropa und der GUS, in: Ifo Schnelldienst 30/1992

Gewerkschaft Textil-Bekleidung, Hauptvorstand, Abtlg. Frauen (1981): Dokumente zu 150 Jahren Frauenarbeit in der Textil- und Bekleidungsindustrie, GTB Düsseldorf

Hausen, Karin (1986): Ideology as Reality. Investigations into Outwork during the 1920s in Germany, paper presented to the E.S.R.C. workshop „The Artisan and Outwork Family”, Essex

- (1997): Arbeiterinnenschutz, Mutterschutz und gesetzliche Krankenversicherung im Deutschen Kaiserreich und in der Weimarer Republik. Zur Funktion von Arbeits- und Sozialrecht für die Normierung und Stabilisierung der Geschlechterverhältnisse, in: Frauen in der Geschichte des Rechts/ Ute Gerhard (Hrsg.), München, 713-743 
Huber, Maria (1999): Eine „nichtliberale“ Demokratie - Rußlands zögernder Weg in die politische Moderne, in: Entwicklung \& Zusammenarbeit 2/1999, S. 36 - 38

International Labour Office, Governing Body (1998): Overview of global developments and Office activities concerning codes of conduct, social labelling and other private sector initiatives addressing labour issues. Geneva

International Labour Office (1998): World Employment Report 1998-1999. Press, Geneva

- (1998a): „Schattenwirtschaft“: ein internationales Phänomen, in: Welt der Arbeit, Nr. 23, 28

Jalusic, Vlasta (1997): Die Geschlechterfrage und die Transformation in Ostmitteleuropa: Kann das Geschlechterparadigma zur „Transformation des Politischen“ beitragen? in: PVS Sonderheft 28/1997, $450 \mathrm{ff}$.

Kessi, Alain (1999): Kosov@/NATO: Ökonomie des Krieges und der Kommunikation, in: com.une.farce (www.copyriot.com/unefarce) Nr. 21999

Köpke, Ronald (1998): Nationaler Wettbewerb und Kooperation. Freie Produktionszonen in Mittelamerika, Münster

Komlosy, Andrea (1997): Textiles Verlagswesen, Hausindustrie und Heimarbeit. Prototypen des informellen Sektors im 18. und 19. Jahrhundert, in: dies. (Hrsg.), Ungeregelt und Unterbezahlt: der informelle Sektor in der Weltwirtschaft, Frankfurt/M., Wien, 63-85

Knapp, Ulla (1984): Frauenarbeit in Deutschland, Band II

Kurz-Scherf, Ingrid (1997): Wenn Arbeit entbehrlich wird - Zur „Krise der Arbeitsgesellschaft“ im ,Zeitalter der Globalisierung“, in: WSI-Mitteilungen, Sonderheft 1997. 41 - 56

Labour Rights in China/ LARIC (1999): No Illusions. Against the Global Cosmetic SA 8000, Hong Kong

Langewiesche, Renate (1998): The EU's eastward enlargement and the social dimension: progress in 1998? In: European Trade Union Yearbook 1998, 305 ff.

Markert, Barbara (1999 a): Jede fünfte PV-Mark geht nach Polen. Passive Veredelung steigt 1998 um 7,3 Prozent auf 11,9 Mrd. DM, in: TextilWirtschaft Nr. 17, 29.04.1999

Meyer, Gert (1998): Transformationskrise in Rußland (I): Ein Land im Rückwärtsgang, in: Informationsbrief Weltwirtschaft \& Entwicklung 11-12/1998

Morgenrath, Birgit/Wick, Ingeborg - Afrika- Süd Aktions-Bündnis (Hrsg., 1996): Die südafrikanische Frauengewerkschaft SEWU. Arbeit im informellen Sektor, Bonn

Musiolek, Bettina (1999): Die Debatte um Verhaltenskodex und Monitoring - eine kritische Zwischenbilanz, in: Gezähmte Modemultis. Verhaltenskodices: ein Modell zur Durchsetzung von Arbeitsrechten? - Eine kritische Bilanz Bettina Musiolek (Hrsg.) Frankfurt a.M.

Potel, Jean-Yves (1999): Der schwindende Charme der EU-Osterweiterung, in: Le Monde Diplomatique, Februar 1999, 4/5

Steilmann, Klaus (1999): Neue Herausforderungen der Verbindung von Ökonomie, Ökologie und Sozialem, in: Gezähmte Modemultis. Verhaltenskodices: ein Modell zur Durchsetzung von Arbeitsrechten?/ Bettina Musiolek (Hrsg.) Frankfurt a.M.

Tesselaar, Annet, FNV Mondiaal (1998): Organizing in the Informal Economy - the role of the trade unions, Amsterdam

UNDP (1998): National Human Development Report. Bulgaria 1998: The State of Transition and Transition of the State. Sofia

Wick, Ingeborg (1998): Frauenarbeit in Freien Exportzonen. Eine Übersicht, in: PROKLA Heft 111, Nr. 2, 235-248

Yanz, Linda/Jeffcot, Bob/Ladd, Deena/Atlin, Joan - Maquila Solidarity Network (Canada) (1999): Policy Options to Improve Standards for Women Garment Workers in Canada and Internationally. Ottawa

Yanz, Linda/Jeffcot, Bob (1999a): Codes of Conduct: from Corporate Responsibility to Social Accountability, www.web.net/ msn, Toronto 